\title{
La ponctuation des lyceens marocains du FLE : Défauts textuels et dispositifs de remediation
}

\author{
${ }^{1}$ Moulay Mohamed TARNAOUI, ${ }^{2}$ Boujghagh HASSAN \\ 1,2 Université IBN ZOHR, Agadir - Maroc \\ Reçu le 21 mars 2018 I Accepté le 21 décembre 2018
}

\begin{abstract}
RÉSUMÉ. Dans le cadre de cette contribution, notre objectif est d'analyser les défauts de ponctuation des lycéens marocains pour répondre à la question suivante : les signes de ponctuation erronés ne contribueraient-ils pas à l'incompréhension et à l'opacité textuelle ? Le support qui servira de base au dépouillement des données est la production écrite. Il est donc crucial de repérer et de classer ces déviations localisées dans les copies des lycéens du FLE. Ces erreurs sont des indices des problèmes d'apprentissage du français chez nos sujets. Notons que cet aspect de la langue est souvent marginalisé par les enseignants bien qu'il contribue à la cohésion textuelle. Les résultats de ce travail ont révélé que la ponctuation reste non maîtrisée et bloque ainsi la compréhension du texte. C'est pourquoi, il sera fructueux de proposer quelques remédiations didactiques en faveur des futurs enseignants.
\end{abstract}

Mots-clés : défauts textuels, français langue étrangère, ponctuation, production écrite

\begin{abstract}
As part of this contribution, our objective is to analyze the punctuation defects of the Moroccan high school students to answer the following question: the punctuation marks mistakes not contribute to the misunderstanding and the textual opacity? The support that will be used as the basis for the data analysis is the written production. It is therefore crucial to identify and classify localized punctuation deviations in FFL high school students' copies. These errors are indicative of the problems of learning French in our subjects. Note that this aspect of language is often marginalized by teachers, although it contributes to textual cohesion. The results revealed that the punctuation remains uncontrolled. That is why it will be fruitful to propose some didactic remedies for future teachers.
\end{abstract}

Keywords : textual flaws, French foreign language, punctuation, written production

auteur correspondant : tarnaouimohamed99@gmail.com

Pour citer cet article (Style APA) : Tarnaoui, M.M. \& Hassan, B. (2018). La ponctuation des lyceens marocains du FLE : Défauts textuels et dispositifs de remediation. Francisola : Revue Indonésienne de la langue et la littérature françaises, 3(2), 141-152. doi: 10.17509/francisola.v3i2.15747 


\section{INTRODUCTION}

Un texte est un ensemble de phrases, mais toute suite de phrases ne constitue pas un texte : entre les phrases d'un même texte, il y a une continuité et le plus souvent une unité de sens (un même thème, un même personnage...). Il se produit aussi nécessairement des ruptures, au bout d'un certain nombre de phrases qui nuisent à la compréhension du message du scripteur. A cet égard, les trois sous- systèmes de la cohésion textuelle à savoir la ponctuation, l'anaphore et les connecteurs sont considérés sous un angle particulier qui consiste à compléter l'approche grammaticale par l'approche discursive (Favart et Chanquoy, 2007).

La tradition grammaticale appréhende longtemps la ponctuation comme l'instrument employé par l'écrit pour restituer des aspects liés à la réalisation orale de la langue. Cette optique venait de la distinction des signes de ponctuation sur la base de paramètre de la longueur des pauses auxquelles ils renvoient (Ferrari et Letizia, 2011). A cet égard, nous voudrions mettre la lumière sur les défauts relatifs à la ponctuation .Le fait que l'écrit reste le modèle de référence comporte des implications sur l'attitude de l'enseignant face aux erreurs linguistiques et textuelles commises par les lycéens. Il est à noter que la correction, la conformité au système de la langue restent un souci majeur du praticien de la classe .

Dans cette veine, la ponctuation est appréhendée selon Grevisse \& Goosse (2008) comme l'ensemble des signes conventionnels servant à montrer, dans l'écrit, des faits de la langue orale comme les pauses et l'intonation, ou à marquer certaines coupures et certains liens logiques. Il est question ainsi d'une conception mixte, alliant le code oral et le code scriptural. Alors que Catach (1980) la saisit comme:

Ensemble de signes visuels d'organisation et de présentation accompagnant le texte écrit, intérieurs au texte et communs au manuscrit et à l'imprimé; la ponctuation comprend plusieurs classes de signes graphiques discrets et formant un système complétant et suppléant l'information alphabétique. (p.20).

Elle est définie aussi comme un ensemble de signes hiérarchisés, dont le rôle est de séparer et d'organiser les différentes parties du discours (Catach, 1994 ; Fayol, 1989). A cet égard, nous soulignons que la ponctuation pose toujours, même implicitement, la question de la compréhension écrite, de la distinction et de l'organisation des parties du discours, de la lisibilité de celui-ci et des effets produits sur les récepteurs (Favriaud, 2001).

Nous rappelons que la notion de « point» comme noyau ponctuationnel, à l'origine même de la terminologie de la ponctuation dans les langues modernes européennes, est née au sein de la latinité : les Grecs ont élaboré des règles de ponctuation, en utilisant notamment des ponctuants polyfonctionnels, pour aider la lecture à haute voix, pour signaler les unités syntaxiques porteuses de sens( Paolo Poccetti, 2011).

Dans la perspective linguistique, la ponctuation fait l'objet de deux tendances antithétiques. D'une part, une approche normative devient dominante. Véronique et Favart (2010) citent Drillon (1991) qui présente dans son ouvrage "une étude du bon usage qu'on fait des signes de ponctuation ». D' autre part, avec les travaux de N. Catach $(1980,1994)$, la ponctuation devient un objet linguistique à proprement parler. Cette spécialiste prend toute la mesure discursive de la ponctuation, en faisant même un carrefour de la textualité, entre écrit et oral, entre syntaxe et énonciation, entre opérations conscientes et coulisses de l'écriture en acte. Elle pose les fondements d'une science de la ponctuation, comme discipline nouvelle et interdisciplinaire au sein de la linguistique.

Dans la même mouvance, Favriaud (2011) a utilisé les concepts de «ponctuation noire » et la «ponctuation blanche». Le premier concerne les marques de ponctuation intérieures au texte et dispose d'une panoplie de signes que les chercheurs peinent à organiser en sous-ensembles stables, accepté de tous par contre le second vise les marques extérieures au texte. Le 
blanc a constamment accompagné l'écriture. Il montre à l'évidence une dimension dans le poème.

Notons que Catach, Tournier, Arrivé, Perrot, Laufer et Védénina parmi d'autres traitent la complexité du système actuel de la ponctuation en posant moult interrogations : qu'est-ce qu'un signe de ponctuation ? En fonction de quoi par exemple peut-on inclure de nouveaux signes dans la liste traditionnelle ? Ne peut-on pas aussi accepter que certaines marques à la façon du blanc de mot ou de page soient des " signes de ponctuation »? N'ont-elles pas été parmi les premières formes de démarcation des unités écrites ? Si l'on autorise que les parenthèses sont des signes de ponctuation et que l'on remarque qu'elles peuvent aider à circonscrire une référence (un nom d'auteur par exemple), pourquoi ne pas considérer logiquement que les notes en bas de page, qui remplissent la même fonction, sont également des marques de ponctuation ? De même, une citation peutêtre signalée par des guillemets (incontestablement signes de ponctuation) ou par un changement de caractères, l'italique le plus souvent. Il existerait donc des signes continus (soulignements, blancs, caractères gras, etc.) et des signes ponctuels ou discontinus. Pourquoi considérerait-on que l'alinéa est une forme de ponctuation et pas le blanc entre les mots ? Si la majuscule de phrase est un signe de ponctuation, la majuscule de mot en est-elle un ? Ces questions demeurent problématiques pour la recherche en linguistique et surtout en didactique du FLE. Elles constituent des zones d'ombres à éclaircir de manière profonde pour que les autres domaines puissent en tirer avantage.

Il ne s'agira pas pour nous de répondre à ces questions cruciales ou de dresser une liste exhaustive de toutes les recherches et de toutes les définitions de la ponctuation dans la mesure où notre cible n'est pas de livrer une recherche sur la ponctuation en elle-même mais uniquement de cerner une notion ayant une importance fondamentale dans la cohésion textuelle.

Le principe essentiel que tout enseignant de français langue seconde/ étrangère devrait systématiquement observer est que écrire est un acte qui s'apprend. De ce fait, la première stratégie que l'on doit concevoir consiste à observer notre action d'enseignement/ apprentissage sous forme de projet pédagogique. L'un des intérêts du projet est de prendre les lycéens tels qu'ils sont et non tels qu'ils sont censés être. Ainsi, à l'issue d'évaluations diagnostiques écrites, les principales difficultés sont répertoriées. Celles-ci constituent ainsi notre base de travail, notre réservoir de données dans le but de concevoir nos séquences didactiques.

L'objectif capital de notre recherche est d'étudier la ponctuation dans la production écrite de nos scripteurs du FLE dans le dessein de vérifier si ce sous- système de la cohésion du texte utilisé par nos lycéens contribue à rendre leur écrit clair et lisible ou au contraire favorise l'opacité pour le lecteur. Il serait crucial de suggérer des pistes didactiques en vue de remédier à cette situation pédagogique qui caractérise l'enseignement du FLE au Maroc.

Dans cette optique, il est primordial de poser les questions suivantes: les lycéens marocains du FLE recourent-ils à une ponctuation adéquate à l'écrit en milieu institutionnel? Quels signes posent-ils réellement problème aux apprenants? L'absence ou la mauvaise exploitation des signes de ponctuation n'engendrent -elles pas un problème de lecture, d'interprétation?

Pour réaliser notre travail, nous avons consciemment limité notre inventaire aux phénomènes d'organisation textuelle les plus saillants concernant la ponctuation. Nous avons pris en considération les signes suivants: alinéa et typographie, le point, le point-virgule, les deux points et les guillemets. C'est pour cela que nous avons ainsi retenu les faits qui sont déficients qui inhibent la cohésion du texte. Celle-ci est appréhendée comme l'ensemble des facteurs qui agissent pour que le texte soit non la simple juxtaposition d'énoncés isolés mais une unité discursive autonome liée à un thème donné. 


\section{MÉTHODE}

L'analyse du Ministère de l'Éducation Nationale du Maroc (2007) concernant le document officiel Les Orientations Pédagogiques et programmes spécifiques à l'enseignement de la langue française dans le cycle secondaire qualifiant montre qu'il n'est plus question de confiner l'apprenant dans cette situation de récepteur inactif étant donné qu'il assume la responsabilité de son apprentissage. L'apprenant, selon la Charte, est placé au centre de l'action pédagogique. En définissant les devoirs et obligations de chacun, elle met au premier plan «l'intérêt de l'apprenant au-dessus de toute considération ». S'inspirant des nouvelles approches, la Charte veut assurer un enseignement actif où le rôle de l'enseignant se limite à celui de guide, d'animateur et de vulgarisateur de la connaissance. Il est à rappeler que le secondaire est appréhendé tel le cycle de la maitrise des langues en l'occurrence la langue française dont la place dans le système éducatif marocain est capitale. L'enseignement de la langue est présenté sous forme de modules avec des supports conçus d'œuvres littéraires.

Le but que nous entreprenons est l'étude du système ponctuationnel du groupe expérimental selon trois niveaux différents de l'apprentissage dans l'intention de vérifier dans un premier temps les déviations qui affectent la production et par conséquent altèrent la compréhension du message. Dans cette perspective, le recours aux données du terrain s'avère essentiel en vue d'optimaliser l'apprentissage.

Dans cette perspective, Le support qui servira de base au dépouillement des données est la production écrite .Nous pourrions faire appel à la typologie discursive. En d'autres termes, nous avons le choix entre le discours narratif, descriptif, argumentatif, injonctif ..., etc. Notons que la production écrite aide à mieux concevoir certains instruments de base et répond notamment aux interrogations suivantes: Comment favoriser la rencontre entre celui qui écrit et le texte? Comment permettre aux apprenants de se constituer progressivement des stratégies qu'ils useront en situation de production? Comment fonctionne la mémoire de l'apprenant dans le fonctionnement de l'information? Comment créer un climat de confiance pour que les élèves puissent s'accommoder des contraintes de l'écrit ? Il est nécessaire que tout futur enseignant s'intéresse et se questionne sur les problèmes que peuvent rencontrer les élèves dans leurs productions écrites. En effet, c'est seulement en examinant des productions des apprenants que les enseignants peuvent réguler leurs activités afin de s'adapter aux difficultés propres à l'apprentissage (Cornaire, 2014).

\subsection{Collecte de données et procédure d'analyse}

Nous avons opté pour la délégation provinciale de Laayoune vu que les études sur cette ville et ses régions sont rarissimes. Aucune étude en FLE n'a été réalisée malgré l'effectif croissant des établissements scolaires au niveau primaire, collégial et secondaire qualifiant. L'échantillon de notre recherche est composé de trois corpus : TCS (Tronc Commun Sciences expérimentales) , 1 AS (année Sciences expérimentales et 2 SVT( Sciences de la Vie et de la Terre) appartenant au lycée qualifiant Mohamed V qui accueille les élèves de toutes les couches sociales. En outre, sa position stratégique et historique le rendent l'un des établissements du secondaire qualifiant de la ville de Laayoune où la population scolaire est importante.

Vus comme un moyen et un support de restitution des connaissances apprises en classe, les écrits des lycéens demeurent un terrain convenable à l'étude des transferts de tout type. Afin de mieux étudier les copies, le moyen adopté est l'analyse des contenus.

En ce qui concerne la procédure que nous avons suivie pour recueillir nos données, nous signalons que nous avons placé les lycéens choisis dans la salle où ils ont l'habitude de travailler. Il s'agit d'un travail individuel : chaque élève a reçu une feuille sur laquelle nous avons précisé le sujet à aborder, la consigne de l'écriture et la durée de l'épreuve. Ensuite, il s'avère opératoire de voir les lieux d'intervention où ces ruptures sont commises par les sujets de notre enquête. Il est question de voir le défaut 
ponctuationnel au niveau de la substitution, de l'omission et de l'adjonction.

Il nous paraît fructueux d'exploiter le type narratif pour les TCS vu qu'il est déjà pratiqué durant le collège et en tronc commun ainsi que le type argumentatif pour la 1ére et la 2éme année bac pour la bonne et la simple raison que le référentiel de l'examen régional du FLE l'impose. De surcroit, le M.E.N a programmé les œuvres intégrales au lycée, la maîtrise de la typologie textuelle devient prépondérante. En prenant en considération les modules programmés, nous avons formulé les sujets de la manière suivante :

Pour les TCS: en te promenant en forêt, tu découvres un passage souterrain. Après bien des hésitations, tu t'engages. Raconte ton aventure en évoquant les impressions et les sentiments que tu as éprouvés.

Pour les 1AS: on dit que les voisins, aujourd'hui, ne sont plus ce qu'ils étaient autrefois : ils aidaient les vieux, les veuves et se comportaient bien avec les enfants. Aujourd'hui, chacun ne pense qu'à ses propres intérêts. Partageriez-vous ce point de vue ? Développez votre réflexion en vous appuyant sur des arguments et des exemples précis.

Pour les 2SVT: les adolescents d'aujourd'hui manquent de respect aux adultes et pensent avoir toujours raison. Partageriez-vous cette opinion? Développez votre conception en vous basant sur des arguments et des exemples précis.

\subsection{Participants}

Rappelons que les élèves, sujets de l'enquête, ont effectué cette tâche sous le contrôle exclusif de leur enseignant afin que l'exercice ne soit pas coupé des tâches scolaires ordinaires. A cet effet, 50 élèves du TCS, 50 élèves de la $1 \mathrm{AS}$ et 50 élèves de la 2SVT ont participé à cette opération. Ce moment consiste à sélectionner fortuitement un prélèvement représentatif de l'ensemble des lycéens qui sont exposés à un savoir, à un savoir-faire en FLE. L'épreuve a lieu le 23 mai 2016.

Le tableau suivant met en relief l'option d'un échantillon représentatif pour chaque niveau d'étude:

Tableau 1. Échantillons requis par niveaux

\begin{tabular}{cccc}
\hline Niveaux & $\begin{array}{c}\text { Population } \\
\text { recensée }\end{array}$ & Echantillons requis & $\%$ \\
\hline TCS & 123 & 50 & 40.65 \\
1 AS & 108 & 50 & 46.29 \\
2 SVT & 110 & 50 & 45.45 \\
\hline
\end{tabular}

\section{RÉSULTATS ET DISCUSSION}

Dans les tableaux suivants, nous exposons les résultats selon la catégorie d'analyse pour chaque échantillon. Il s'agit essentiellement des défauts de ponctuation. L'accent est par conséquent mis fondamentalement sur les productions

Tableau 2. Répartition des anomalies ponctuationnelles selon niveau d'étude

\begin{tabular}{cccc}
\hline Catégorie/Niveau d'étude & TCS & 1 AS & 2 SVT \\
\hline Défauts de ponctuation & 737 & 367 & 627 \\
Moyenne (par copie) & 14,74 & 7,34 & 12,54 \\
\hline
\end{tabular}

Ce tableau indique la situation des défauts textuels de la ponctuation de la population touchée par l'enquête. Ainsi, la moyenne d'erreurs en TCS est de 14,74 par copie. Pour les productions écrites en 1 AS, déviantes à forts coefficients d'occurrences et qui sont des indices des problèmes d'apprentissage du français chez nos sujets.

Ainsi, le nombre de déviations textuelles relatives à la ponctuation est porté sur le tableau suivant : 
déviations à l'antipode, ces dernières tendent à la baisse en 1 AS.

Les lycéens produisent leur écrit en faisant entorse à la norme ponctuationnelle.
Ce qui explique l'importance et la motivation chez les lycéens pour les examens régionaux. Ce qui éprouverait que les efforts déployés visent l'évaluation sommative.

Tableau 3. Répartition des défauts de la ponctuation selon les niveaux scolaires

\begin{tabular}{ccccccc}
\hline $\begin{array}{c}\text { Signes de la } \\
\text { ponctuation défectueux }\end{array}$ & $\begin{array}{c}\text { Occurrences } \\
\text { en TCS }\end{array}$ & $\mathbf{\%}$ & $\begin{array}{c}\text { Occurrences en } \\
\text { 1ère bac }\end{array}$ & $\mathbf{\%}$ & $\begin{array}{c}\text { Occurrences } \\
\text { en 2SVT }\end{array}$ & $\%$ \\
\hline Alinéa et typographie & 101 & 13,72 & 27 & 7,35 & 81 & 12,9 \\
Point & 352 & 47,76 & 171 & 46,59 & 234 & 37 \\
La virgule & 223 & 30,25 & 118 & 32,15 & 290 & 46,25 \\
Le point-virgule & 0 & 0 & 0 & 0 & 0 & 0 \\
Les deux points et les & 61 & 8,27 & 51 & 13,91 & 22 & 3,85 \\
$\begin{array}{c}\text { guillemets } \\
\text { Total des occurrences }\end{array}$ & 737 & 100 & 367 & 100 & 627 & 100 \\
\hline
\end{tabular}

En ce qui concerne les erreurs de la ponctuation, il paraît que les lycéens confondent totalement le code écrit et le code oral. Les traces de ce dernier foisonnent dans la performance rédactionnelle des lycéens. D'une année à une autre, les dysfonctionnements persistent. Nous constatons que les élèves du TCS et de 2SVT ont commis plus que le double de déviations ponctuationnelles par rapport aux élèves de 1AS. Le point et la virgule constituent la part $\mathrm{du}$ lion en matière de déviations ponctuationnelles. A cela s'ajoute la répartition du texte en paragraphe qui crée des problèmes aux apprenants. Ces derniers ne différencient plus à quel moment utiliser un paragraphe et à quel autre employer un texte. Il est en de même pour la virgule et le point. Quant aux deux points, ils sont rarement exploités et le point-virgule n'est plus utilisé puisque nous n'avons repéré aucune occurrence dans le corpus constitué de 150 copies.

Ces anomalies constatées dans ce corpus seraient liées d'une part à l'évolution cognitive de l'apprenant et de sa prise de conscience des faits linguistiques. Cela rejoint Fayol (1997 cité par Favart \& Passerault (2000) qui voit que ce sont bien les structures cognitives gouvernant la production qui constituent le principal déterminant de la fréquence et de la nature de la ponctuation. En effet, le type narratif est totalement différent du type argumentatif. Nous avons l'impression qu'en TCS, les élèves n'accordent pas une grande importance au code scriptural et à la spécificité du texte narratif. Ils ne veillent ni sur la forme ni le contenu. Ils ne sont pas guidés par aucune consigne dans le but de leur laisser cette marge de liberté.

Les signes ponctuationnels restent certainement obligatoires à la fois pour structurer un texte et pour apporter des éléments d'information supplémentaires au sens d'une phrase. Il ne faut donc pas négliger la ponctuation, car elle facilite tout aussi bien la lecture que la compréhension et elle est fondamentale lors de l'expression écrite. L'étude de Fayol (1989) présente deux qualités, la première est de situer la ponctuation dans le cadre général des contraintes inhérentes à la linéarité de la production langagière et de suggérer quelques arguments expérimentaux sur le fonctionnement textuel de la ponctuation en production et en compréhension. Son analyse met en relief la préciosité de la mise en place de ce système.

Concernant le code oral, les individus qui parlent partagent la même situation : ils n'ont pas constamment besoin de préciser qui ils sont, où ils sont et l'instant où ils se parlent ; alors qu'à l'écrit, toutes ces précisions doivent être arborées pour que le récepteur comprenne de façon facile l'écrit. L'usage incohérent des signes ponctuationnels dans les productions écrites de nos lycéens pourrait être l'indicateur de la non- maturation syntaxique et sémantique. Sur ce plan, il est essentiel de noter que dans de nombreux ouvrages de la grammaire, les 
pages de la ponctuation ont souvent été placées dans les chapitres consacrés aux phénomènes de l'oralité.

La norme en matière de ponctuation n'est plus respectée par certains apprenants. Les uns ne distinguent plus entre texte et paragraphe, d'autres ne recourent plus au point et à la virgule, d'autres encore confondent les signes de ponctuation et rendent ainsi le texte difficile à comprendre comme le montre la copie suivante extraite du corpus de la 1 ère du bac:

j'ai lu dans un journal un paragraphe qui parle sur les enfants, mais ce qui m'interaissait c'était la question suivante faut-il dire ou cacher la vérité aux enfants? et ils était écris que la plus parts des parents préfère cacher la vérité de leur enfants car il ne veulent pas leur déranger avec des choses qui vont pas leurs aider à cette âge et aussi à cause d'éducation que les parent préfère à leur enfants par exemple il y a des parents qui veulent que leur enfants seront consantrer juste sur ce qu' il va leur aider à leur étude lu qu' il y avait la moitié des parents qui préfère dire la vérité à leur enfants parce que quand ils découvrent la vérité il veut être choqué et il vont pas faire encore confiance à leur parents, donc ces derniers préfère de ne pas cacher la vérité pour moi je pense $q u^{\prime}$ il faut être serieux avec les enfants et leur dire la vérité car comme ça quand il devient grand sil seront capable de leur vie et ils vont étudié quelque choses qu'il savent avant. (Copie d'un élève de la 1AS).

L'exemple de la copie révèle que les règles relatives à la ponctuation ne sont pas respectées. Les élèves transgressent les signes conventionnels de la ponctuation. Nous remarquons que Le point qui marque seul une pause plus longue que tous les signes tels la virgule, point-virgule, etc. n'est pas respecté. La règle stipule qu'on le met à la fin de toutes les phrases, de toutes les périodes dont le sens est complet.

Dès le départ, les alinéas et les paragraphes ne sont pas marqués. L'absence $\mathrm{du}$ point, du point-virgule, la virgule rend la production incohérente. L'accès au sens paraît difficile et par conséquent, le lecteur ne parvient pas à déchiffrer le sens et la signification $\mathrm{du}$ texte. Pour réaliser cette tâche fastidieuse, il lui faut du temps, de l'effort et de la concentration pour déterminer la véracité du message. La segmentation du texte devient un impératif à respecter .Dans l'optique où nous travaillons, nous citons un autre exemple parmi d'autres qui élucide la même situation en TCS :

un jour, je décidais avec mes amis de faire une sortie à la forêt pour découvrir un passage souterrain pendant notre chemin à la forêt un homme de 40 ans portait des vêtments sale et déchirée me demandait de lui donner l'argent ou il tuait moi mais mes amis lui attaquaient avec les pierres puis nous avions s'enfuir quand nous arrivions à la forêt, nous partîmes pour découvrir le passage soudain un lion attaquait nous, nous étions très peur que le lion dévorait nous, tout-à-coup un pêcheur lui attaquait avec son pistolé après nous remercions le pêcheur, puis nous avion terminer notre chemin soudain l'un de mes amis lui mordait un serpent, puis je téléphonais l'ambilance pour lui traverser à l'hôpitale après nous revenions à la maison. Il était une journée inoubliable grâce à ses aventures.

Comme la copie précédente, il semble que la ponctuation pose un véritable problème pour les élèves. Ils segmentent aléatoirement leur texte. Globalement il est question d'une sous-ponctuation .En dépit des efforts déployés, les élèves souffrent de la non maturation syntaxique vu que cette dernière dépare la production de l'apprenant qui croit réaliser des performances positives. L'absence du point crée la difficulté et rend le message véhiculé incompréhensible ou opaque .Le lecteur doit faire de grands efforts pour décoder le message. Il est à remarquer que les élèves ne relisent pas leur texte pour rectifier certaines maladresses comme le montre les deux textes de notre échantillon.

De surcroit, ces élèves ne distinguent pas les signes de ponctuation de manière claire et participent ipso facto à la malformation textuelle. La sous-ponctuation ne facilite pas la compréhension du message véhiculé par les apprenants. A titre d'exemple, ils ne séparent pas entre le paragraphe et le texte, entre le point, le pointvirgule et la virgule. La confusion règne entre les signes et nécessite une intervention didactique. Parallèlement, les élèves de $2 S V T$, vivent la même situation comme en témoigne la copie suivante: 
aujourd'hui on trouve des gens qui sont avec la proverbe et les gens avec le deuxième le premier dit qu'il y a une grande différence enre les voisins d'autrefois et les voisins d'aujourd'hui, et le deuxième proverbe dit " avant d'avoir la maison il faut choisir les voisins " c'est un proverbe poupulaire et les gens qui sont avec le deuxième prouverbe disent que les voisins sont importants car ils peuvent donner beaucoup d'aider à ces voisins comme aider les vieus et les voisin qui sont tomber dans une crise ou dans un problem avec quelqu'un mais il y a des gens qui pensent que tous ces problemes aujourd'hui ou veut pas les trouver des solutions car ces jour -là on trouve beaucoup de phénoménes entre les voisins comme le racisme et le mensonge et le vol finalement, je crois qu'il faut protéger les principe les gens d'autrefois car ils ont beaucoup des aspects positifs sur notre famille et notre société.

Les analyses et les réflexions que nous proposons répondent à un double objectif. Il s'agit, tout d'abord, de prouver les incohérences liées à la ponctuation et qui créent des difficultés pour le lecteur de la production écrite et ensuite de suggérer des pistes didactiques en vue de remédier à cette situation pédagogique qui caractérise l'enseignement du FLE au Maroc.

Ceci nous conduit à proposer une hypothèse selon laquelle les emplois de la virgule répondent à deux types de régularités modélisables: des régularités formelles de types syntaxiques et des régularités textuelles. A cet effet, il s'avère que la ponctuation est plus une affaire de message que d'énoncé. C'est pour cette raison qu'il est impérieux de combiner les deux notions pour déterminer les conditions dans lesquelles l'emploi des signes doit être réglé. Ainsi, dans ce cadre, lorsqu'elle concerne l'énoncé, la virgule est syntaxique ; quand elle se charge du message, elle est textuelle ou macro-syntaxique répondant à la structuration sémantico-pragmatique. Dans le premier exemple ci-dessous, il est question de l'énoncé (grammaire de la phrase/microsyntaxe) et dans le second, il s'agit du message.

Premier exemple:

chaque être humain a ses problèmes, et il a besoin de les confier a quelqu'un pour se rassurer psychiquement néanmoins est-ce qu'il choisissait la bonne personne pour lui confier (copie de la 1AS).

Second exemple :

un jour je décidais avec mes amis de faire une sortie à la forêt pour découvrir un passage souterrain pendant notre chemin à la forêt, un homme de 40 ans portait des vêtments sale et déchirée me demandait de lui donner l'argent ou il tuait moi mais mes amis lui attaquaient avec les pierres, puis nous avions s'enfuir, quand nous arrivions à la forêt, nous partîmes pour découvrir le passage. Soudain un lion attaquait nous, nous étions très peur que le lion dévorait nous tout-à-coup un pêcheur lui attaquait avec son pistolé après nous remercions le pêcheur puis nous avion terminer notre chemin soudain, l'un de mes amis lui mordait un serpent puis je téléphonais l'ambilance pour lui traverser à l'hôpitale après nous revenions à la maison. Il était une journée inoubliable grâce à ses aventures (copie du TCS).

La distribution des résultats montre que la ponctuation demeure un obstacle pour les élèves de notre enquête. Nombreuses sont les copies qui illustrent la sous-ponctuation. Ceci est valable pour le TC, 1 AS et la 2 SVT. Ainsi, les structures textuelles demeurent non maîtrisées. Le système d'appropriation de nos sujets présente des failles et des lacunes; d'où le problème d'une bonne segmentation, de saisie du texte et de son interprétation. C'est pourquoi l'enseignement devrait donner une grande place à la ponctuation et la grammaire de texte, en particulier les anaphores et les connecteurs qui participent activement à la cohésion du texte.

Soulignons que les signes de ponctuation sont produits dès les premières années du primaire (Fabre, 1989). Le premier signe à apparaître est le point qui rythme la succession des contenus produits selon une planification locale, réalisée pas à pas, grâce à la stratégie des connaissances rapportées. La virgule est utilisée un peu plus tardivement, à partir du CE2. Cependant, ces élèves de 8-9 ans ne différencient pas la virgule et le point comme introduisant un degré de rupture différent. Il en résulte une utilisation ambivalente, voire concurrentielle de ces deux signes (Simon, 1966). Les points sont produits au niveau macrostructural, tandis 
que la description microstructurale des activités est parfois rythmée par la virgule. « À 13-14 ans, la ponctuation est acquise en tant que système. À cet âge-là, les apprenants tiennent compte de leur lecteur dans le guidage, et la segmentation est illustrée à un niveau plus microstructurel du texte. » (Paolacci, Rossi-Gensane, 2014, p. 120).

Quant à Favart et Passerault (2000), ils mettent en rapport l'évolution dans l'usage $\mathrm{du}$ point et de la virgule avec l'acquisition d'une planification globale et hiérarchisée des écrits, effective au CM2 dans le récit en images. Cette adéquation entre mode de planification du texte et utilisation des signes est également attestée par Favart et Passerault (2009) dans les descriptions produites par des élèves de l'école primaire. Ainsi, les apprenants, quel que soit leur niveau scolaire, réalisent une planification globale de leurs textes. La ponctuation est progressivement acquise par les enfants en tant que système hiérarchiquement structuré. Les considérations en question prouvent les évolutions parallèles de la planification et de la maîtrise du système, et les difficultés de leur gestion simultanée chez les enfants.

La ponctuation n'est plus enseignée actuellement ni au collège ni au lycée; d'où les lacunes constatées dans les productions écrites de nos sujets. « La ponctuation en tant que telle est absente du cursus scolaire dans le primaire et le premier cycle du secondaire. Son enseignement est laissé à l'initiative des enseignants comme s'il s'agit là de conventions dont l'importance est secondaire » (Chami, 1987, p. 124).Le volume horaire consacrée à la grammaire de la phrase et surtout la grammaire du texte ne permet pas d'attaquer ces ruptures au niveau de l'écrit. La majorité des élèves ignorent même les signes de ponctuation.

A ce propos, Chami (1987) déduit que la charge fonctionnelle de la ponctuation est absente dans les copies des élèves marocains. Dans ce contexte, il explique :

Les nombreuses perturbations relevées dans les copies des élèves marocains de notre échantillon à propos de la ponctuation traduisent une certaine indiscipline dans leur pensée provenant sans doute d'une maîtrise imparfaite de la langue française. Cela donne à leurs écrits l'allure d'une écriture souvent incohérente et dont le lecteur a souvent $\mathrm{du}$ mal à repérer la segmentation véritable qu'ils veulent donner à leurs phrases (p. 124).

$\mathrm{Vu}$ la fréquence de ces anomalies, il est important de les étudier puisque la prise en charge de ces constructions déviantes en classe pourra optimaliser l'enseignement / apprentissage. Pour cette raison, l'investissement dans le domaine didactique nous semble prioritaire et fructueux. Notre focus sera ciblé sur la cohésion textuelle pour aider les apprenants à rédiger dans des conditions optimales. Dans le dessein de réaliser cette tâche, il est fructueux de montrer aux lycéens le rôle des anaphores, de la ponctuation et les connecteurs dans la cohésion et la cohérence du texte.

La nature et l'emplacement des signes seront le reflet des deux niveaux de planification du récit : le niveau global, ou macrostructural et le niveau local, ou microstructural. Dans le cas de la planification $\mathrm{du}$ récit, la production écrite serait guidée par une structure conceptuelle hiérarchique. Favart \& Passerault (2000) citent Favart \&Passerault (1996) avancent à ce propos:

Ainsi, la structure du texte se hiérarchise selon deux niveaux (global et local). Cette hiérarchisation permet, au niveau local, une expansion des contenus, qui se traduit par un allongement significatif des protocoles. Elle permet, à ce même niveau local, l'apparition de modes spécifiques de mises en relation dont les traces sont repérables dans la mise en texte (p.201).

\subsection{Quelques \\ didactiques}

remédiations

Il convient donc d'aider les enseignants à assurer ou à renforcer les bases dans leurs pratiques personnelles, en travaillant deux aspects fondamentaux de l'expression écrite : grammaire de phrase et grammaire de texte, de façon à contribuer à l'amélioration de la situation actuelle. La notion de grammaire de texte ne leur est pourtant pas tout à fait étrangère et il est curieux de constater que des annotations 
comme ponctuation, paragraphe, liaison, omission, répétition, pronom ne sont pas regroupés lors de la correction sous la même catégorie de cohérence ou de cohésion selon les échelles du texte.

Il serait intéressant et bénéfique d'étudier les trois fonctions de la ponctuation en classe dès le début de l'année à savoir: fonctions prosodique, syntaxique et sémantique. Ces trois fonctions sont étroitement liées. En effet, les pauses en lecture orale sont d'autant plus longues que les signes présentent des ruptures syntaxiques fortes. En outre, la valeur des signes utilisés est directement en fonction de la force de la liaison existant entre les propositions. Ainsi, les signes ponctuationnels ont un rôle qui structure la production écrite.

Quant à 1' aspect didactique de la ponctuation, des interrogations restent posées: comment enseigner les signes ponctuationnels? Faut-il enseigner tous ces signes? Il est crucial de distinguer entre les savoirs scolaires et les savoirs savants. C'est dans cette optique que Lemaître (1995) a précisé :

Ces perspectives nouvelles, cette vue d'ensemble des marques qui structurent un texte, de la ponctuation traditionnelle aux procédés de mise en page, peuvent permettre à l'enseignant de ne pas dissocier certains apprentissages mais les débats de détail restent l'affaire de spécialistes. Quel intérêt y aurait-il par exemple à inclure dans la liste des signes de ponctuation proposée aux élèves l'apostrophe, le trait d'union, le blanc de mots ? Ont-ils à y gagner dans leurs apprentissages orthographiques ? Les savoirs scolaires doivent faciliter les apprentissages et non pas forcément calquer les savoirs savants, surtout lorsque ceux-ci ne font pas encore l'unanimité des spécialistes... La question de l'inventaire des signes, dans une perspective élargie, appartient donc sans doute au domaine des savoirs savants dont on n'entrevoit pas encore les possibles retombées didactiques(p.165).

L'établissement des exercices correctifs en matière de ponctuation a pour objectif fondamental d'aider les apprenants à mieux utiliser les marques de cohésion textuelle en FLE. Dans ce sens, une meilleure exploitation des différents signes par le praticien ne peut qu'actualiser des optima d'apprentissage. L'enseignant fera appel à des ouvrages classiques tels La bonne ponctuation de Doppagne (1978), Traité de la ponctuation française de Drillon (1991) ..., etc., ceux que nous avons cités dans cet article ou il recourrait à l'internet pour enrichir son cours sur le plan théorique et pratique.

Dans la même optique, l'approche de Paolacci et Rossi-Gensane (2014) nous paraît importante du moment que l'enseignant ou le futur enseignant pourrait y recourir. Cette démarche consiste à étudier le système de ponctuation autrement en suivant trois étapes : la première, l'élève peut être incité à segmenter le texte par des barres sans s'interroger sur la sorte de signe de ponctuation à insérer à l'endroit concerné ; la seconde, l'élève est conduit à appliquer une segmentation à l'aide de connecteurs qui peuvent être remplacés par des signes de ponctuation comme les signes de ponctuation forte; la troisième, Les élèves les plus avancés sont invités à employer une panoplie de signes de ponctuation selon leurs besoins dont les différentes fonctions seront travaillées en classe.

Sur le même plan et pour aider les élèves qui souffrent d'une segmentation opératoire de leur texte, nous proposerions au futur praticien de s'inspirer de la méthode exploitée par Colognesi et Deschepper (2014) dans une étude sur le savoir des élèves en ponctuation surtout la grille à la page 47 .

Si on force l'apprenant à ne produire que le français standard, la norme, il aura de la peine à rédiger faute de moyens linguistiques adéquats. A cet effet, on part des formes déviantes, on localise les lacunes et on tente de les combler par des exercices appropriés. Dans ce sens, on peut parler de grammaire corrective ou d'une pédagogie compensatoire.

\section{CONCLUSION}

Cette investigation a montré que la majorité des apprenants de notre enquête ne maîtrisent pas les signes de ponctuation du 
moment qu'ils sont dépourvus de moyens linguistiques de base. Grosso modo, une réflexion didactique plus approfondie reste à construire en matière de ponctuation. Sa difficulté, ses liens partiels avec l'oral et parfois l'absence de normes suffisamment reconnues rendent son apprentissage peu évident.

Le problème reste d'élaborer un modèle descriptif cohérent et fortement intégrateur à partir de la forme, de la fonction et de la place des signes dans l'ensemble du texte. Cette marque de cohérence textuelle est encore actuellement un sujet d'étude largement ouvert à la controverse. Nous avons vérifié la place de la ponctuation dans la production écrite du groupe expérimental pour la bonne et simple raison qu'elle affecte la cohésion des productions écrites de nos scripteurs. De surcroit, le lecteur aura des problèmes à décoder et à saisir le sens ou l'interprétation des textes des scripteurs vu que les énoncés construits par nos lycéens sont mal segmentés.

Le déficit phrastique et textuel de la ponctuation des lycéens de l'enquête a posé la question de la communication écrite, de la distinction et de l'organisation des parties du discours, de la lisibilité de celui-ci et des effets produits sur les récepteurs. L'obstacle demeure présent quant à la maitrise de la cohésion en français. Les apprenants ne sont pas dotés de stratégies pour contrôler et vérifier leurs énoncés. La spontanéité demeure une caractéristique capitale chez eux, rares sont les lycéens qui révisent leurs copies avant de les rendre au professeur. Ainsi, l'élaboration d'une batterie d'exercices relatifs à la cohérence et à la cohésion s'avère opératoire et fructueuse.

\section{REMERCIEMENTS}

Je tiens à remercier plus particulièrement A. Amsidder, qui s'est toujours montré à l'écoute. Il a constamment le cœur sur la main. De même, le directeur du laboratoire LARLANCO Mokhtar Mouhal pour son soutien constant. Un grand merci à mon ami, Kennate Mohamed, qui a pris le pli de m'appuyer continuellement durant la rédaction de la thèse et de cet article. Je le remercie et je souhaite de mon cœur qu'il termine son ouvrage «Takat: question de vie ou de mort?»

\section{RÉFÉRENCES}

Catach , N. (éd.) (1980). La ponctuation. Langue française $\mathrm{n} \circ 45$. Paris: Larousse.

Catach, N. (1994). La ponctuation, Paris. Presses Universitaires de France, Coll. 'Que sais-je ?'

Chami, M. (1987). L'enseignement du français au Maroc: diagnostic des difficultés et implications didactiques. Casablanca, Najah el Jadida.

Colognesi, S et Deschepper, C. (2014). Le savoir des élèves en ponctuation. Analyse des interactions et propositions didactiques ", Le français aujourd'hui 2014/4 ( $\mathrm{n}^{\circ} 187$ ), p. 43-55. Repéré à DOI 10.3917/lfa.187.0043

Cornaire, C. (2014). La production écrite. Paris, Clé International.

Grevisse, Maurice et André Goosse (2008), Le bon usage: grammaire française, $14 \mathrm{e}$ édition, Bruxelles et Louvain-laNeuve, Éditions De Boeck et Duculot.

Doppagne A. (1978). La bonne ponctuation. Paris : Duculot.

Drillon J. (1991). Traité de la ponctuation française, Paris : Gallimard, Coll. 'Tel'.

Fabre C. (1989). «Les débuts de la ponctuation au CP»,Études de linguistique appliquées, 73, 59-70.

Favart M, Passerault J-M. (2000). Aspects fonctionnels du point et de la virgule dans l'évolution de la planification $\mathrm{du}$ récit écrit. Enfance, tome 53, $\mathrm{n}^{\circ} 2$, 2000. pp. 187-205; doi : 10.3406/enfan.2000.3176 Repéré à http://www.persee.fr/doc/enfan_0013 -7545_2000_num_53_2_3176

Favart, M et Chanquoy, L. (2007). Les marques de cohésion comme outils privilégiés de la textualisation: une comparaison entre élèves de CM2 et adultes experts. Langue française 155 p. 51-68

Favart M. \& Passerault J.-M. (2009). Acquisition of relations between the conceptual and the linguistic 
dimensions of linearization in descriptive text composition in grades five to nine: A comparison with oral production. British Journal of Educational Psychology 79, 107-130.

Favriaud M. (2011). Plurisystème ponctuationnel, dimension, intensité des signes et architecturation du texte poétique. Langue Française 172. p.83- 97

Fayol M. (1989). Une approche psycholinguistique de la ponctuation. Étude en production et en compréhension. Langue française, 81, 21-39.

Ferrari, A et Letiza L.(2011). Les emplois de la virgule en italien contemporain. De la perspective phono-syntaxique à la perspective textuelle. Langue Française 172. pp.53-68.

Grevisse, Maurice \& André Goosse. (2008). Le bon usage: grammaire française, 14e édition, Bruxelles et Louvain-laNeuve, Éditions De Boeck et Duculot.

Lemaître, B. (1995). La ponctuation : un savoir enseignable? Enseigné ?
SPIRALE - Revue de Recherches en Éducation - N¹5 pp 161-195.

Ministère de l'Éducation Nationale du Maroc. (2007). Orientations pédagogiques et programmes spécifiques à l'enseignement de la langue française dans le cycle secondaire qualifiant. Maroc.

Paolacci, V Rossi-Gensane, N. (2014). Ponctuation et écrits d'élèves : Une conception différente de la phrase pour enseigner la ponctuation autrement. Le français aujourd'hui 2014/4 (n 187), p. 115-125. Repéré à DOI 10.3917/lfa.187.0115

Poccetti, P. (2011). La réflexion autour de la ponctuation dans l'Antiquité grécolatine. Langue Française 172. p19-35.

Paolacci, V. et Favart M. (2010). Traitement des marques de cohésion par les jeunes scripteurs: l'utilisation de la ponctuation et des connecteurs à l'entrée en sixième. Approche linguistique, cognitive et didactique. Langages 1/2010 ( $\mathrm{n}^{\circ}$ 177), p. 113-128. Vol.21. Verlag Peter Lang $\mathrm{GmbH}$, Frankfurt am Main. 